\title{
L'HOMME L'Homme
}

Revue française d'anthropologie

$170 \mid 2004$

Espèces d'objets

\section{Éditorial}

"La règle de la boîte de conserve"

Jean Jamin

\section{OpenEdition}

\section{Journals}

Édition électronique

URL : http://journals.openedition.org/lhomme/24774

DOI : 10.4000//homme.24774

ISSN : 1953-8103

Éditeur

Éditions de l'EHESS

\section{Édition imprimée}

Date de publication : 1 juin 2004

Pagination : 7-10

ISSN : 0439-4216

Référence électronique

Jean Jamin, «Éditorial », L'Homme [En ligne], 170 | 2004, mis en ligne le 01 janvier 2006, consulté le 24 septembre 2020. URL : http://journals.openedition.org/lhomme/24774 ; DOI : https://doi.org/10.4000/ Ihomme.24774 


\title{
Éditorial
}

\section{"La règle de la boîte de conserve"}

\begin{abstract}
?
EST PROBABLEMENT en songeant au livre de Georges Perec, Espèces d'espaces ${ }^{1}$, que Jean Bazin eut l'idée du titre de ce volume auquel, peu de temps avant sa disparition, il avait fixé l'objectif de sinon renouveler du moins modifier l'approche et l'analyse anthropologiques de toutes ces choses qui nous entourent, nous meublent, nous charment ou nous déplaisent, que l'on garde ou jette, que l'on étudie, contemple ou dédaigne - pour la plupart choses d'un usage oublié ou redéfini, voire recyclé, ou choses carrément hors d'usage, comme sil fallait que, pour être dignes d'attention esthétique, elles ne soient plus en état de marche... Donc choses usées, traces, archives de l'activité humaine, parfois dotées d'une valeur testimoniale, patrimoniale ou emblématique et que, mises sous vitrine ou en réserve, élevées en monuments, disposées sur un étal, dressées sur le manteau d'une cheminée, enfouies dans un coffre, on désigne généralement par un mot-joker tel que celui d'objets - fussent-ils "objets de peu " comme une bouteille de cidre ou une réderie de la Somme, ou objets disons métaphoriques comme une relique, une figure de proue satanique, une voix gravée sur disque (sorte d' "existant invisible " ${ }^{2}$ ) ou, grandioses et désormais froids, comme des hauts fourneaux lorrains, ou encore objets esthétiques et cultuels de sociétés autres, dont le qualificatif de primitifs qui longtemps servit à les désigner leur avait en quelque sorte dénié la qualité d'ouvres et, pour finir, ôté toute dignité artistique, ravalés ainsi au rang de simples objets de curiosité que, jusqu'au début du XX siècle, on regardait pourtant d'un oeil distrait, condescendant, plutôt amusé.
\end{abstract}

Percer l'apparente opacité des objets ou au contraire interroger leur trop grande transparence au moyen des méthodes et de la description ethnographiques, tel était l'enjeu, entre le trait et le pointillé, le galbe et la matière, la forme et la fonction, voire

1. Georges Perec, Espèces d'espaces, Paris, Galilée, 2000 (1 $1^{\mathrm{re}}$ éd. 1974).

2. Rossana Dalmonte, "Voix", in Jean-Jacques Nattiez, ed., Musiques. Une encyclopédie pour le XXI siècle. 1. Musiques du XXe siècle, Arles, Actes Sud-Cité de la musique, 2003 : 446. 
entre le vide et le trop-plein ("Ce qui compte dans le vase, c'est le vide du milieu", avait écrit Jean-Paul Sartre sur le bandeau publicitaire de L'Être et le Néant) - là encore à la façon de Georges Perec qui, "sobligeant à voir platement", réussissait ce tour de force de nous désorienter rien qu'en décrivant de la manière la plus évidente, commune et terne, une rue de Paris et ses trottoirs. À la différence des surréalistes qui, souvent, cherchèrent à dépayser les objets, Jean Bazin y voyait déjà matière à dépaysement: il suffisait d'inventorier leurs usages, d'ajuster le regard et de se forcer à détailler non seulement leurs contours mais leurs amoncellements, emplacements et "placements ", intentionnels ou non, les uns par rapport aux autres.

La poursuite et l'approfondissement de sa réflexion sur la description en anthropologie ${ }^{3}$ (en cet art ingrat, négligé il y a peu, aujourd'hui reconsidéré $e^{4}$, Jean Bazin était passé maître comme en témoigne son article devenu rare sur "Le roi sans visage " que nous reproduisons en ouverture de cette livraison), tout autant que les discussions et controverses auxquelles donna lieu le projet de création du musée dit "du quai Branly" en 1998, et dont, avec Alban Bensa, il fut l'un des participants les plus clairvoyants - et des plus incisifs ${ }^{5}$-, l'avaient amené à repenser la question de l'objet ethnographique (ou pas), esthétique (ou minable, voire sordide), fonctionnel (ou absurde, imaginaire même $e^{6}$, et à tester ce qu'il appelait, en mémoire de Marcel Mauss, "la règle de la boîte de conserve" 7 .

Ce thème de l'objet, de ses espèces et espaces, sétait imposé à Jean Bazin au cours de conversations animées que nous ềmes fréquemment, que ce soit au sein du conseil de rédaction de L'Homme, à l'issue de ses séminaires de l'École des hautes études en sciences sociales ou encore, plus banalement, autour d'un verre ou d'un plat (souvent les deux) dans un bistrot. Elles étaient en partie motivées et nourries par les soubresauts que connaissait, depuis le début des années 1990, le musée de l'Homme, à l'avenir incertain, aux collections ethnographiques sous-exposées, au budget mité à l'image de nombre des tissus qui y étaient conservés. Bien qu'il n'eût jamais appartenu à cette institution, Jean Bazin en était devenu un observateur perspicace; il en avait très vite aperçu les impasses, lesquelles se repéraient notamment dans les galeries et les réserves

3. Cf. Jean Bazin, «Interpréter ou décrire. Notes critiques sur la connaissance anthropologique», in Jacques Revel \& Nathan Wachtel, eds, Une École pour les sciences sociales, Paris, Cerf-Éditions de l'EHESS, 1996 : 401-420.

4. Cf. Giorgio Blundo \& Jean-Pierre Olivier de Sardan, eds, Pratiques de la description, Paris, Éditions de l'EHESS, 2003; cf. également, pour l'apport de Jean Bazin à la "théorie de la description" en anthropologie, l'article de Marie Gaille-Nikodimov, "Comment réduire l'altérité?", Critique, 2004, 680-681: 121-124, notamment.

5. Cf. Jean Bazin \& Alban Bensa, "À propos d'un musée flou ", Le Monde du 19 avril 2000.

6. Tels le couteau de Lichtenberg, le crâne de Blaise Pascal enfant, ou le fer à repasser de Man Ray dont la semelle est garnie de clous..., exemples qu'affectionnait particulièrement Jean Bazin.

7. Cf. Jean Bazin, «N'importe quoi ", in Marc-Olivier Gonseth, Jacques Hainard \& Roland Khaer, eds, Le Musée cannibale, Neuchâtel, Musée d'ethnographie, 2002:273-287 (postface de Michèle de La Pradelle). Jean Bazin rappelle que, dans les Instructions sommaires pour les collecteurs d'objets ethnographiques (brochure rédigée en mai 1931 par Michel Leiris à partir des cours de Marcel Mauss à l'Institut d'ethnologie), il est fait allusion à cette boutade de Mauss : "Une boîte de conserve, par exemple, caractérise mieux nos sociétés que le bijou le plus somptueux ou que le timbre le plus rare. Il ne faut donc pas craindre de recueillir les choses même les plus humbles et les plus méprisées [...] En fouillant un tas d'ordures, on peut reconstituer toute la vie d'une société."

\section{Espèces d'objets}


où les collections d'objets ethnographiques étaient toujours grevées de la conception lourdement positiviste qui seétait développée (et figée) depuis le milieu des années 1930. Mais il n'entendait pas se borner à cette critique institutionnelle ni fonder sa recherche sur les seuls aspects ethnologiques et muséologiques de l'objet, préférant l'escapade épistémologique à "l'escalade ontologique" (pour reprendre une formule de Nathalie Heinich et Jean-Marie Schaeffer ${ }^{8}$ ): celle qu'il vit à l'ouvre dans l'opposition qui se faisait soudain jour (alors qu'elle semblait révolue) entre, d'un côté, les "esthètes" de la forme et, de l'autre, les «savants" de la fonction, chacune des deux parties senfermant dans une conception essentialiste et, ce faisant, disciplinaire de son objet : histoire de l'art versus anthropologie culturelle.

Les avancées théoriques de certaines sciences humaines - outre l'ethnologie: la préhistoire, l'archéologie, la sociologie de l'art, des techniques, de la consommation, la philosophie de l'esthétique, etc. - qui ont affaire à de l'objet, auraient dî̀ toutefois conduire à d'importants remaniements et corrections dans le tracé des frontières cognitives et épistémiques de chacune, et mettre en évidence si ce n'est en abîme non seulement la polysémie ou la multifonctionnalité de l'objet, mais son devenir-autre - en un mot son historicité, ou ce que Jean Bazin appelait "sa mise en situation".

Un objet n'est pas la simple expression ou concrétion d'une relation; son existence matérielle pas plus que sa fonction ne préjugent de sa destinée culturelle. Déjà, dans les années 1950, à propos d'une catégorie particulière d'objets (ceux relevant de l'ethnomusicologie où le rapport forme-fonction semble prédéterminé et peu sujet à manipulation), André Schaeffner ${ }^{9}$ avait montré qu'un instrument ne se limite pas seulement à sa fonction et que, bien plus, le lien souvent se relàche entre ce à quoi il paraissait voué et les formes successives de sa musicalité. Tout se passe comme si les musiciens s'employaient à faire sonner différemment leur instrument, comme si la fantaisie humaine, ou rien qu'une lubie, risquait de "toujours faire se renverser l'instrument": ainsi de ces flùtes à bouche devenues nasales qu'on trouve en Afrique noire; ainsi des torsions (par exemple la trompette coudée de Dizzy Gillespie) et distorsions (" dirty notes") que les musiciens de jazz ont fait subir aux instruments de facture classique. En somme, l'objet ne se réduit pas à la chose, celle-ci n'étant jamais que la totalité virtuelle de ses "objectivations" concurrentes ou successives.

Jean Bazin conçut donc un argument qui fut adressé à des chercheurs dont les intérêts et les travaux sattachaient à dépasser la querelle "des anciens et des modernes" - mais sans quion pût vraiment départager les uns des autres -, qui sévissait alors dans une partie de la communauté anthropologique française, focalisée sur des problèmes de muséologie, c'est-à-dire de collecte, de conservation et d'exposition, bien que ce qui était ou ce qui devait être collecté, conservé et exposé - c'est-à-dire de l'objet ethnographique - ne fût guère interrogé sur le fond. Il s'agissait pour lui de reprendre le problème à sa racine et de ne négliger aucune des pistes ou traces que dessinait l'objet, surtout pas celles quil pouvait laisser dans nos propres sociétés, même si ces dernières

8. Cf. Nathalie Heinich \& Jean-Marie Schaeffer, Art, création, fiction. Entre sociologie et philosophie, Nîmes, Éd. Jacqueline Chambon, 2004 : 9; infra, l'article de Jean-Marie Schaeffer.

9. Cf. André Schaeffner, Variations sur la musique, Paris, Fayard, 1998. 
produisent de l'objet jusqu'à l'écourement au point d'en inventer, comme par traitement homéopathique, des formes jetables... Aussi, à la fin de cet argument, Jean Bazin en appelait-il à la description et analyse rigoureuses d'un objet ou d'un ensemble d'objets, en faisant apparaître les différents usages, présentations et accumulations possibles tant dans la société productrice que dans la société réceptrice ou conservatrice, et ce en prenant en compte aussi bien le point de vue des "consommateurs" ou utilisateurs disons communs que celui des institutions et des disciplines qui se sont donné pour tâche de les sauvegarder et de les étudier.

Les articles réunis dans ce volume ne peuvent qu'imparfaitement refléter le projet qu'avait eu Jean Bazin. Si, dans leurs grandes lignes, il prit connaissance des textes aujourd'hui publiés, il ne put en suivre - comme il avait l'habitude de le faire, crayon en main - l'édition et l'appariemment. Manquent surtout, cruellement, l'introduction qu'il avait projeté d'écrire, et sa propre contribution qu'il aurait souhaité centrer sur le kono, cet "innommable objet" que ni les femmes ni les enfants ni les étrangers ne pouvaient voir mais que le jeune ethnographe Michel Leiris, pendant une halte de la Mission Dakar-Djibouti en septembre 1931, avait dérobé dans un village bambara du Mali : sorte de "cochon de lait" fait de boue et de sang séchés (trace des sacrifices de volailles dont il avait été le réceptacle) et qui, au début des années 1980, fut exposé comme un des cent chefs-d'auvre du musée de l'Homme dans un centre culturel consacré aux arts africains à New York. Quelque temps plus tôt, il avait été mis en valeur au Musée d'ethnographie de Neuchâtel, au moment de l'exposition "Collections passion" ${ }^{10}$; après l'inauguration, il avait même donné lieu à une petite manifestation de la part d'étudiants et enseignants en anthropologie, qui, sur l'air des lampions, chantèrent nuitamment "On a le kono!" dans les rues de la ville... Parfaite illustration, en vérité, de cette indétermination culturelle de l'objet dès lors qu'il est extrait de son contexte et qu'il est devenu "hors d'usage"! Comme le note Michèle de La Pradelle, commentant l'article inachevé de Jean Bazin sur lequel il travaillait lorsque la mort le faucha un après-midi de décembre 2001, article humoristiquement intitulé "N'importe quoi" et qu'il tenait à proposer à un nouveau catalogue du Musée d'ethnograpie de Neuchâtel avant de s'atteler au kono pour L'Homme (comme si la boucle ne devait jamais se boucler): "Avec ces choses [les objets ethnographiques], ces ouvres humaines préservées de la destruction, du retour à la poussière, on refait une autre cuvre, une cuvre au carré en quelque sorte..." ${ }^{11}$

Par la force des circonstances, ce volume de L'Homme devait prendre la forme d'un hommage. Et c'est en accord avec les auteurs que nous le dédions à la mémoire de Jean Bazin, qui a toujours su donner à ses recherches et enseignements un véritable esprit d'aventure et d'ouverture intellectuelles, un esprit d'escapade anthropologique auquel voudrait céder l'examen des espèces d'objets ici présentées.

Jean Jamin

10. Cf. Jacques Hainard \& Roland Khaer, eds, Collections passion, Neuchâtel, Musée d'ethnographie, 1982.

11. Michèle de La Pradelle, postface à «N'importe quoi » de Jean Bazin, art. cit., 2002: 287. 